\title{
Study on structural recovery of graphite irradiated with swift heavy
}

\section{ions at high temperature}

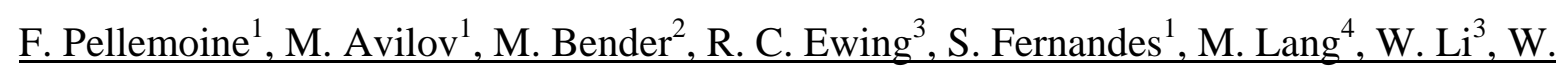

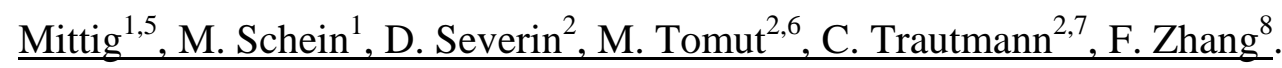

1. Facility for Rare Isotope Beams, Michigan State University, East Lansing MI 48824, USA

2. Dept. of Materials Research, GSI Helmholtzzentrum für Schwerionenforschung,

Planckstr. 1, Darmstadt 64291, Germany

3. Dept. of Geological Sciences, Stanford University, Stanford, CA 94305-2115, USA

4. Dept. of Nuclear Engineering, University of Tennessee, Knoxville, Knoxville, TN 37996-2300, USA

5. National Superconducting Cyclotron Lab, Michigan State University, East Lansing MI 48824-1321, United States

6. Laboratory of Magnetism and Superconductivity, National Institute for Materials Physics NIMP, Bucharest, Romania

7. Dept. of Materials Science, Technische Universität Darmstadt, Darmstadt, Germany 8. Department of Earth and Environmental Sciences, University of Michigan, Ann Arbor, MI 48109, USA

\begin{abstract}
Thin graphite foils bombarded with an intense high-energy (8.6 MeV/u) gold beam reaching fluences up to $1 \times 10^{15}$ ions $/ \mathrm{cm}^{2}$ lead to swelling and electrical resistivity changes. As shown earlier, these effects are diminished with increasing irradiation temperature. The work reported here extends the investigation of beam induced changes of these samples by structural analysis using synchrotron X-ray diffraction and transmission electron microscope.
\end{abstract}


A nearly complete recovery from swelling at irradiation temperatures above about $1500{ }^{\circ} \mathrm{C}$ is identified.

\section{INTRODUCTION}

The realization of a production target for the new generation of high-energy high-intensity heavy ion beam facilities such as the rare isotope production at the Facility for Rare Isotope Beams (FRIB) [1] in the USA and at the Facility for Antiproton and Ion Research (FAIR) [2] in Germany is challenging. The target material has to withstand the combined effects of high power density beams generating thermo-mechanical stress as well as radiation damage leading to structural modifications and changes of thermo-mechanical properties. All these effects will limit the reliable function and the lifetime of the targets.

In collaboration within the FRIB and FAIR project, thin graphite foils were irradiated at the GSI UNILAC accelerator with an intense $8.6 \mathrm{MeV} / \mathrm{u}$ gold beam at fluences up to $1 \times 10^{15}$ ions $/ \mathrm{cm}^{2}$. The samples showed swelling and an increase of the electrical resistivity. These beam-induced changes were observed to diminish with increasing irradiation temperature [3].

This paper presents an extended investigation of part of these irradiated graphite samples, using synchrotron X-ray diffraction (XRD) and High Resolution Transmission Electron Microscope (HRTEM). Measurements of the unit-cell parameter $\mathrm{c}$ and d spacing provide new insights into graphite performance under extreme ion beam exposure. Applying a 8.6 MeV/u Au beam provides an electronic energy loss value of $~ 20 \mathrm{keV} / \mathrm{nm}$ [4], which is higher than the future FRIB beams (energy loss between 0.04 and $6 \mathrm{keV} / \mathrm{nm}$ ). The target material is thus tested under a harsher energy density deposition than in future applied conditions. 


\section{EXPERIMENTAL PROCEDURE}

The experimental set-up is defined in more detailed description in reference [3]. As samples we used thin foils of purified polycrystalline isotropic graphite (MERSEN grade 2320) with a nominal thickness of $75 \mu \mathrm{m}$. During the irradiation with $8.6 \mathrm{MeV} / \mathrm{u} \mathrm{Au}$ ions, the electric current for ohmic heating was adjusted to the desired irradiation temperature by applying

currents up to $36 \mathrm{~A}$. A total of four samples were irradiated at different fluences up to $1 \times 10^{15}$ ions $/ \mathrm{cm}^{2}$ and temperatures up to $1535^{\circ} \mathrm{C}$. The parameters of the different samples and irradiations are presented in TABLE I.

The samples were characterized before and after irradiation by synchrotron X-Ray Diffraction measurements (XRD) performed at the Advanced Photon Source (Lawrence Berkeley Natl. Laboratory), using high energy x-ray (30 keV). The x-ray wavelength was $0.4132 \AA$.

High Resolution Transmission Electron Microscope (HRTEM) analysis was performed with a JEOL 3011 electron microscope at the University of Michigan. For TEM inspection, the irradiated samples were crushed and deposited on a carbon film supported by a copper TEM grid. Gold particles were intentionally deposited on top of the ground graphite specimens in order to calibrate the scale bars. During the TEM observations, the electron current density was kept as low as possible $\left(0.1\right.$ to $\left.1 \mathrm{~A} / \mathrm{cm}^{2}\right)$ to minimize electron-beam-induced microstructural changes.

\section{RESULTS AND DISCUSSION}

\section{X-Ray Diffraction analysis}

The diffraction patterns were recorded for graphite samples irradiated at different fluences and temperatures are shown in Figure 1. Comparing the diffraction pattern of the pristine and the sample irradiated at $385{ }^{\circ} \mathrm{C}$ reveals a pronounced broadening of the (002) diffraction maximum. Peak broadening is in general attributed to a reduction of grain size [5] and/or 3 
build-up of micro-strain in the sample [6]. Moreover, the broadening of the (002) peak is accompanied by a shift to a smaller angle (TABLE I), indicative for an increase of the dspacing. In contrast, the (110) diffraction maximum is insensitive to the ion irradiation showing within our experimental accuracy neither peak broadening and nor a shifts (TABLE I) which indicates no significant change of the in-plane lattice parameter.

With increasing irradiation temperature, the (002) diffraction maximum shifts back towards its original two theta value (pristine sample) concomitant with a reduction in width, revealing in situ defect annealing. The increase of the (002) peak intensity at higher temperature is ascribed to re-crystallization of the polycrystalline graphite. At the maximum irradiation temperature of $1535^{\circ} \mathrm{C}$, the sample has mostly recovered its original structure even after irradiation to the maximum fluence of $1 \times 10^{15}$ ions $/ \mathrm{cm}^{2}$. However, a small peak shift and broadening remain detectable.

\section{HRTEM analysis}

A HRTEM image of a graphite sample irradiated at $1525^{\circ} \mathrm{C}$ (fluence $1 \times 10^{14}$ ions $/ \mathrm{cm}^{2}$ ) together with the corresponding Fast Fourier Transform (FFT) analysis is presented in Figure 2. Variations in lattice parameter are subtle for samples irradiated at different temperatures. Therefore, instead of directly measuring the d-spacing in the HRTEM images, gold particles with the known d-spacing were used to calibrate precisely the d-spacing of graphite. From the FFT image, the d-spacing of graphite (111) was determined by the constant $R^{*} d$ relation where $\mathrm{R}$ is the radius of a ring in the diffraction pattern. In a FFT image, a vector pointing from (0000) to a diffraction spot represents the corresponding crystal direction in the imaging plane. The lattice spacing of (111) gold is $\mathrm{d}_{\mathrm{Au} 111}=2.35 \AA$, the lattice spacing of (111) carbon is derived by 
$\mathrm{d}_{\mathrm{C} 111}=\mathrm{d}_{\mathrm{Au} 111} \times\left(\frac{\mathrm{R}_{\mathrm{Au} 111}}{\mathrm{R}_{\mathrm{C} 111}}\right)$.

The deduced values of the d-spacing of graphite are reported in TABLE I for each sample.

From HRTEM and synchrotron XRD analyses, we inferred the lattice parameter changes from d-spacing values of peak (002) and peak (111) which is relative to the changes in c-axis, with respect to the pristine sample as a function of irradiation temperature. FIGURE 3 shows the relative lattice parameter change as a function of irradiation temperature for two fluences. Both XRD and HRTEM analysis revealed a consistent lattice expansion of 3-4\% for an irradiation temperature up to about $1000^{\circ} \mathrm{C}$. Further increase of the sample temperature during irradiation results in less radiation damage. This in-situ annealing effect is evident in the XRD and HRTEM data and seems to act at both fluences tested. Extrapolating this result, we expect that irradiations performed at or above a temperature of $1900{ }^{\circ} \mathrm{C}$ avoid beaminduced structural changes of graphite.

Defect annealing is also supported by results from post-irradiation investigations of electrical resistance [3], thermal diffusivity [7] and Young's modulus [8] properties of heavyion irradiated graphite that had similar pristine properties and irradiation conditions as the ones used in this study. For the irradiation of similar polycrystalline fine-grain graphite with fast neutrons, it was also observed that defect annealing associated with partial property recovery occurs at irradiation temperatures above $1000^{\circ} \mathrm{C}$ [9].

\section{CONCLUSION}

Gold ions of 8.6 MeV/u kinetic energy and high electronic energy loss (20 keV/nm) were used to irradiate polycrystalline graphite (MERSEN Grade 2320) at different fluences and 
irradiation temperatures. HRTEM and XRD analyses were performed and consistently identify an increase of the lattice parameter at irradiation temperatures up to about $1000^{\circ} \mathrm{C}$. For irradiations at $1535^{\circ} \mathrm{C}$, the lattice parameter expansion is less pronounced indicating defect annealing effects during irradiation. Target operation at such high temperature obviously mitigates radiation damage and make this type of graphite a suitable material for use in a high-power production target designed for rare isotope beam production at FRIB and FAIR.

\section{ACKNOWLEDGMENT}

The authors gratefully acknowledge technical support during synchrotron XRD at the Advanced Photon Source of Lawrence Berkeley National Laboratory. The Advanced Light Source is supported by the Director, Office of Science, Office of Basic Energy Sciences, of the U.S. Department of Energy under Contract No. DE-AC02-05CH11231. The TEM and XRD analysis was completed at the University of Michigan and supported by the Energy Frontier Research Center Materials Science of Actinides funded by the U.S. Department of Energy, Office of Science, Office of Basic Energy Sciences (DE-SC0001089).

This material is based upon work supported by the U.S. Department of Energy Office of Science under Cooperative Agreement DE-SC0000661, the State of Michigan and Michigan State University. Michigan State University designs and establishes FRIB as a DOE Office of Science National User Facility in support of the mission of the Office of Nuclear Physics. 


\section{REFERENCES}

1. D.J. Morrissey, J.Phys.: Conference Series 267 (2011) 012001.

2. H. Geissel, H. Weick., M. Winkler, G. Münzenberg, V. Chichkine, M. Yavor, et al., Nucl. Instr. and Meth. B 204 (2003) 71-85.

3. S. Fernandes, F. Pellemoine, M. Tomut, M. Avilov, M. Bender, M. Boulesteix, M. Krause, W. Mittig, M. Schein, D. Severin, C. Trautmann, Nucl. Instr. and Meth. B 314 (2013) 125-129.

4. J. F. Ziegler, M. D. Ziegler and J. P. Biersack, Nucl. Instr. And Meth., B268, 18181823 (2010) and www.srim.org/. SRIM Version: SRIM-2013.00

5. M. Boccanfuso, A. Benyagoub, M. Toulemonde, C. Trautmann, K. Schwartz, Ch. Dufour, Nucl. Instr. and Meth. B 175-177 (2001) 590-593.

6. C.L. Tracy, M. Lang, J.M. Pray, F.X. Zhang, D. Popov, C. Park, C. Trautmann, M. Bender, D. Severin, V.A. Skuratov, and R.C. Ewing, Nature Communications 6, 6133 (2015).

7. N. Horny, M. Chirtoc, M. Tomut, M. Avilov, M. Bender, W. Mittig, F. Pellemoine, M. Schein, D. Severin, and C. Trautmann, GSI Scientific Report 2011, PNI-MR-16, 414. 8. M. Tomut, H. Weick, M. Avilov, M. Bender, M. Gleim, C. Karagiannis, I. Manika, J. Maniks, W. Mittig, F. Pellemoine, M. Schein, K. Schwartz, D. Severin, C. Trautmann, and R. Zabels., GSI Scientific Report 2011, PHN-NUSTAR-FRS-23, 165.

9. B. T. Kelly, Irradiation behavior of fine-grain graphites, Fusion Technology, Vol. 16 (1989) p. 96-103. 
TABLE I. Irradiation parameters of graphite grade 2320 samples irradiated during a previous experiment (Ref [3]) and used here in further XRD and HRTEM analyses. The sample name denotes the heating current (in Amp) applied during irradiation. d-spacing extracted from the Synchrotron XRD pattern (peak (002) and peak (110)) performed at Advanced Photon Source (Lawrence Berkeley Natl. Laboratory). d-spacing extracted from HRTEM investigation (peak (111)) performed at University of Michigan. From these values, the relative expansion is inferred and compared to the pristine sample.

\begin{tabular}{|c|c|c|c|c|c|}
\hline Samples & Pristine & $1 \mathrm{~A}$ & $19 \mathrm{~A}$ & $35 \mathrm{~A}$ & $36 \mathrm{~A}$ \\
\hline Mean flux (ions $/ \mathrm{cm}^{2} \mathrm{~s}$ ) & & $3.8 \times 10^{10}$ & $4.5 \times 10^{10}$ & $4.2 \times 10^{10}$ & $5.6 \times 10^{10}$ \\
\hline Fluence (ions $/ \mathrm{cm}^{2}$ ) & & $1 \times 10^{14}$ & $1 \times 10^{14}$ & $1 \times 10^{14}$ & $1 \times 10^{15}$ \\
\hline $\begin{array}{l}\text { Irradiation temperature } \\
\qquad\left({ }^{\circ} \mathrm{C}\right)\end{array}$ & & $385 \pm 40$ & $910 \pm 150$ & $1525 \pm 95$ & $1535 \pm 275$ \\
\hline d-spacing $(002)(\AA)$ & 3.4197 & 3.5415 & 3.5330 & 3.4666 & 3.4642 \\
\hline $\begin{array}{l}\text { Relative expansion of d- } \\
\text { spacing }(002)(\%)\end{array}$ & & $3.57 \pm 0.50$ & $3.32 \pm 0.47$ & $1.37 \pm 0.59$ & $1.30 \pm 0.19$ \\
\hline d-spacing (110) $(\AA)$ & 1.2438 & 1.2443 & 1.2426 & 1.2471 & 1.2427 \\
\hline Relative expansion of d- & & $0.04 \pm 0.01$ & $-0.10 \pm 0.01$ & $0.26 \pm 0.15$ & $-0.09 \pm 0.01$ \\
\hline d-spacing (111) $(\AA)$ & 3.47 & 3.58 & & 3.50 & \\
\hline $\begin{array}{l}\text { Relative expansion of d- } \\
\text { spacing }(111)(\%)\end{array}$ & & $3.17 \pm 0.32$ & & $0.86 \pm 0.09$ & \\
\hline
\end{tabular}


List of figures

FIGURE 1. Synchrotron XRD patterns of graphite samples irradiated at different temperatures and fluences. The bottom pattern is the pristine sample for reference and the top pattern is the sample irradiated to the highest fluence $\left(1 \times 10^{15}\right.$ ions $\left./ \mathrm{cm}^{2}\right)$; the three patterns inbetween are data from samples irradiated to the same fluence $\left(1 \times 10^{14}\right.$ ions $\left./ \mathrm{cm}^{2}\right)$ but at different temperatures.

FIGURE 2. HRTEM investigation of sample irradiated at $1525^{\circ} \mathrm{C}$ up to a fluence of $1 \times 10^{14}$ ions $/ \mathrm{cm}^{2}$. Left - HRTEM image of carbon sample (stripped lattices) and gold nanoparticules (black spots) deposited for calibrating the d-spacing of the carbon. Right corresponding Fast Fourier Transform (FFT) image; the two green dotted rings, which intersects most of the diffraction maxima with the same radius, are a guide to the eyes.

FIGURE 3. Lattice parameter expansion (\%), representative of changes in c-axis, relative to pristine graphite sample at different irradiation temperatures and two fluences inferred from HRTEM and XRD analyses. 


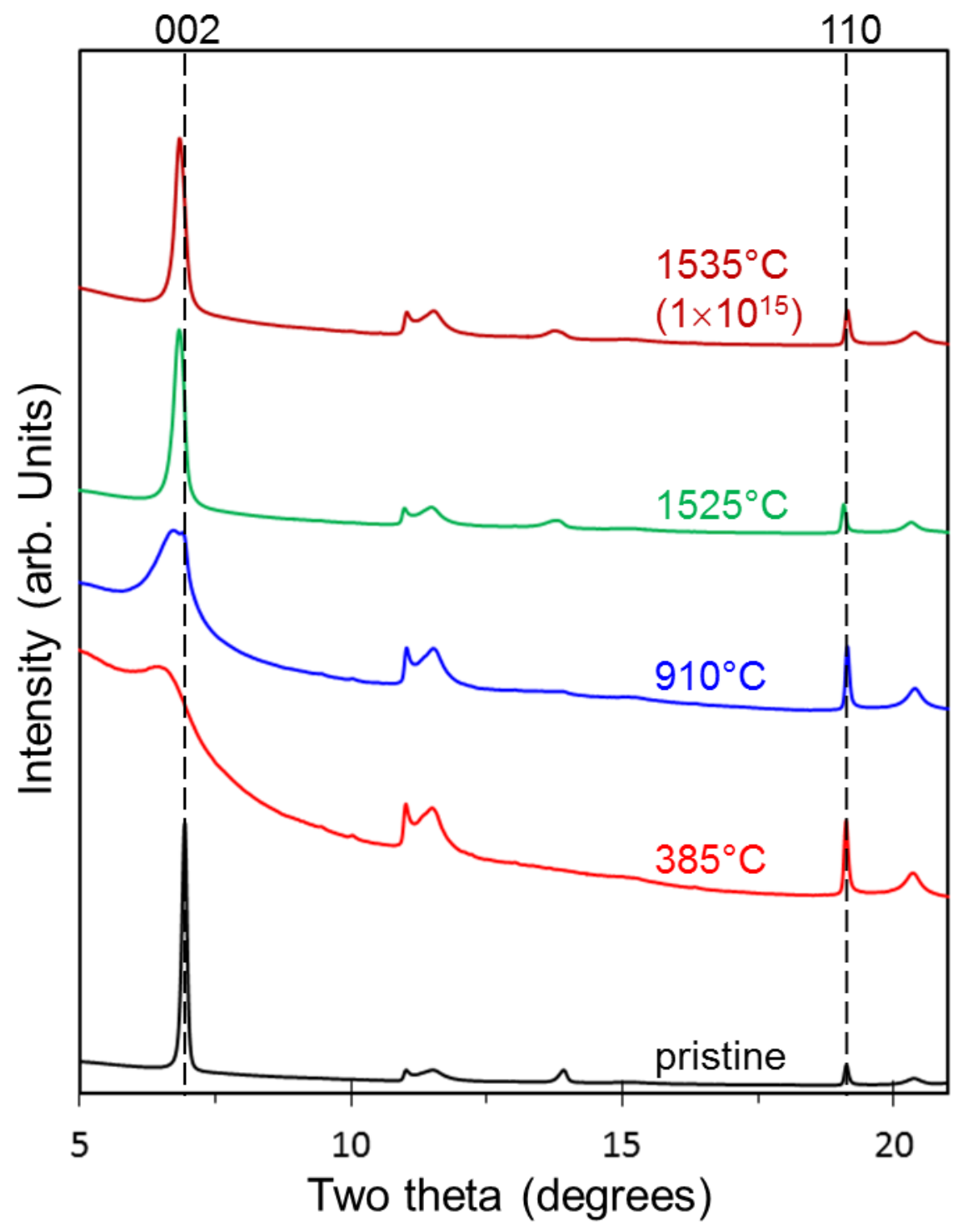

FIGURE 1 

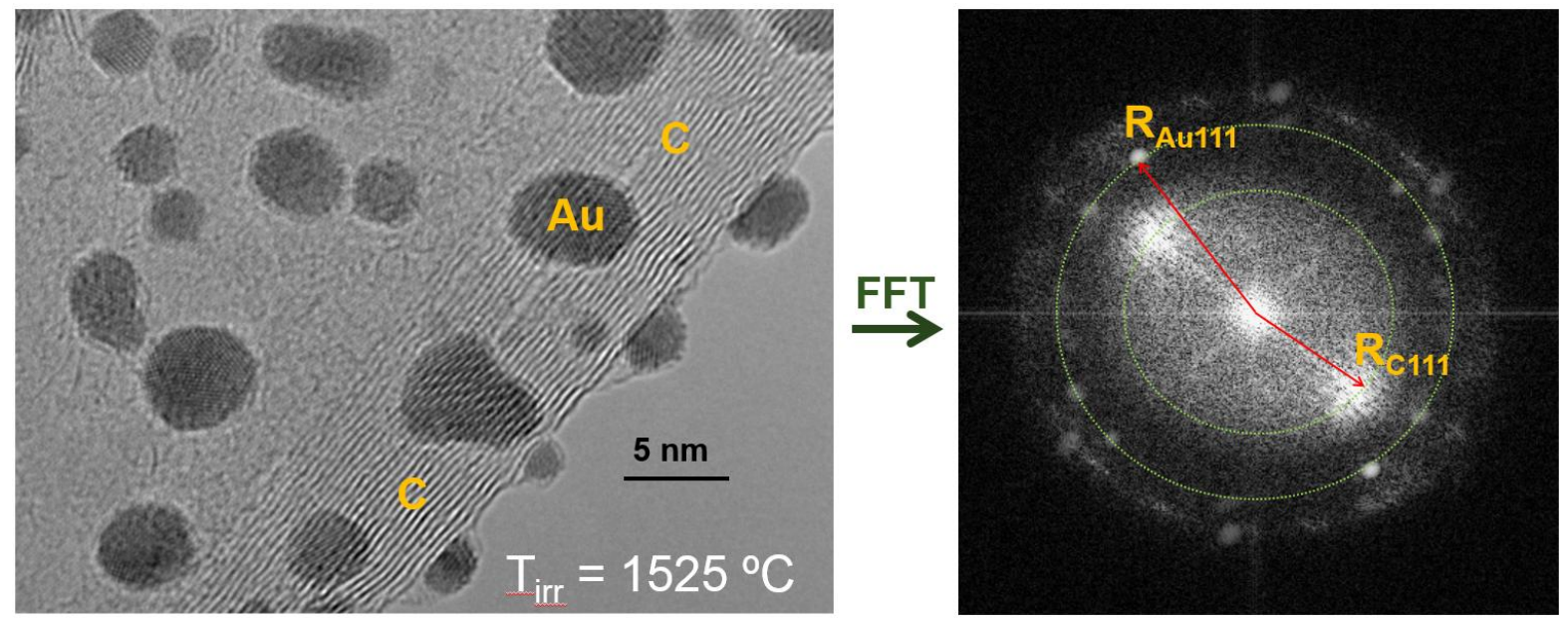

FIGURE 2 


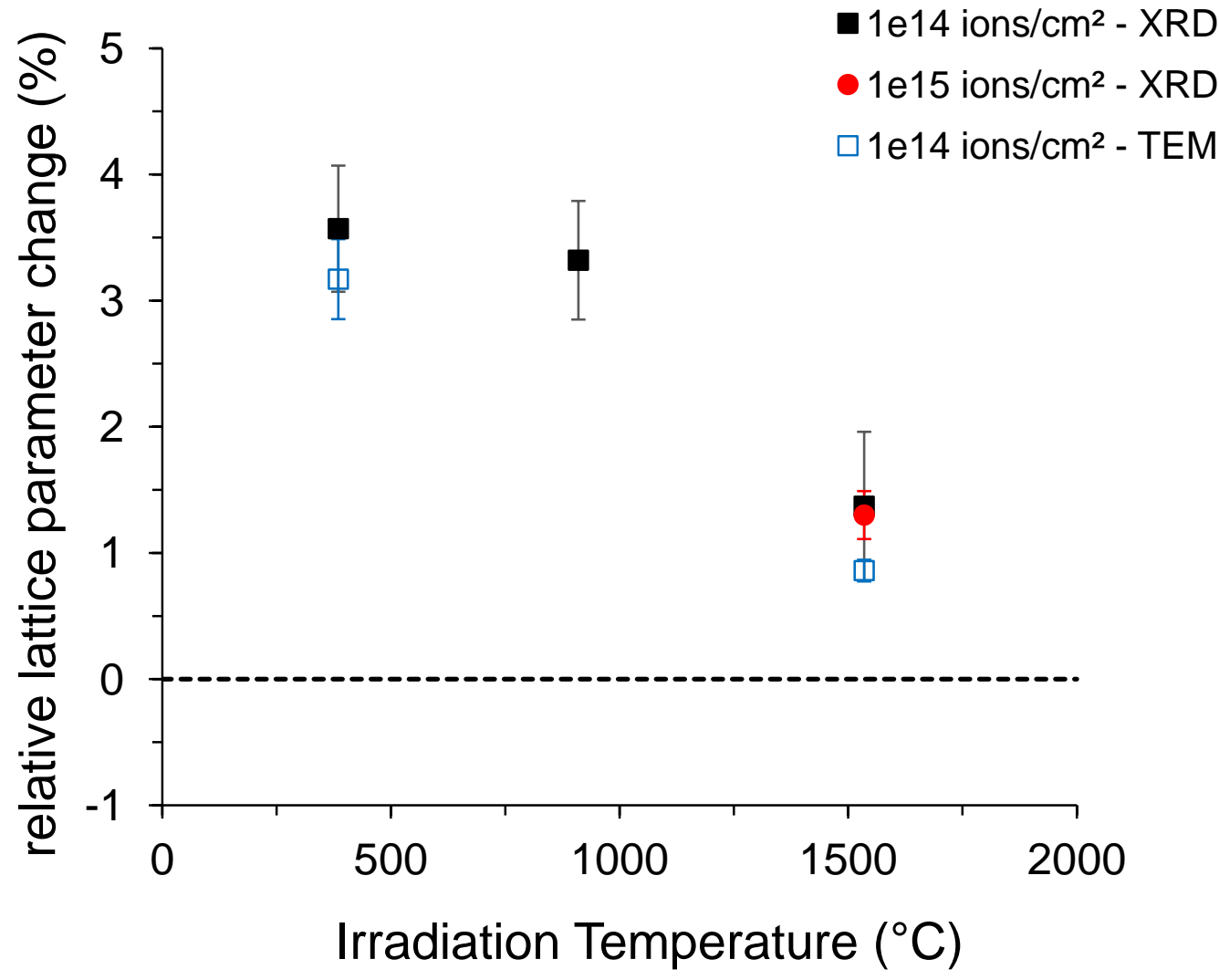

FIGURE 3 